\title{
Fermilab
}

Managed by Fermi Research Alliance, LLC for the U.S. Department of Energy Office of Science

\section{Irradiation Induced Failure Analysis of NuMI Target}

Sujit Bidhar

Dec 11, 2019

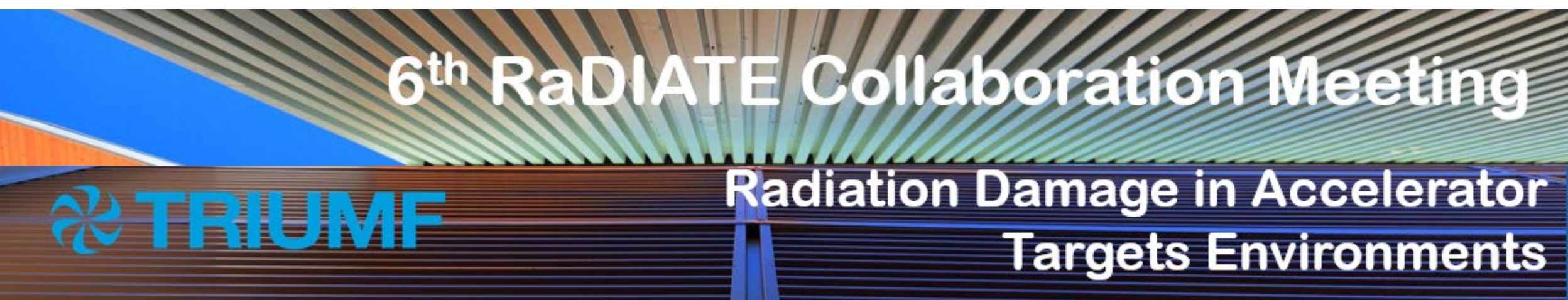

6th RaDIATE Collaboration Meeting

This manuscript has been authored by Fermi Research Alliance, LLC under Contract No. DE-AC02-07CH11359 with the U.S. Department of Energy, Office of 


\section{Outline}

- Background NTO2 Target fin

- Fin material properties and response under irradiation

- Swelling empirical formula

- Finite element implementation and results

- Failure analysis 


\section{Background- NT02 Target Fin}
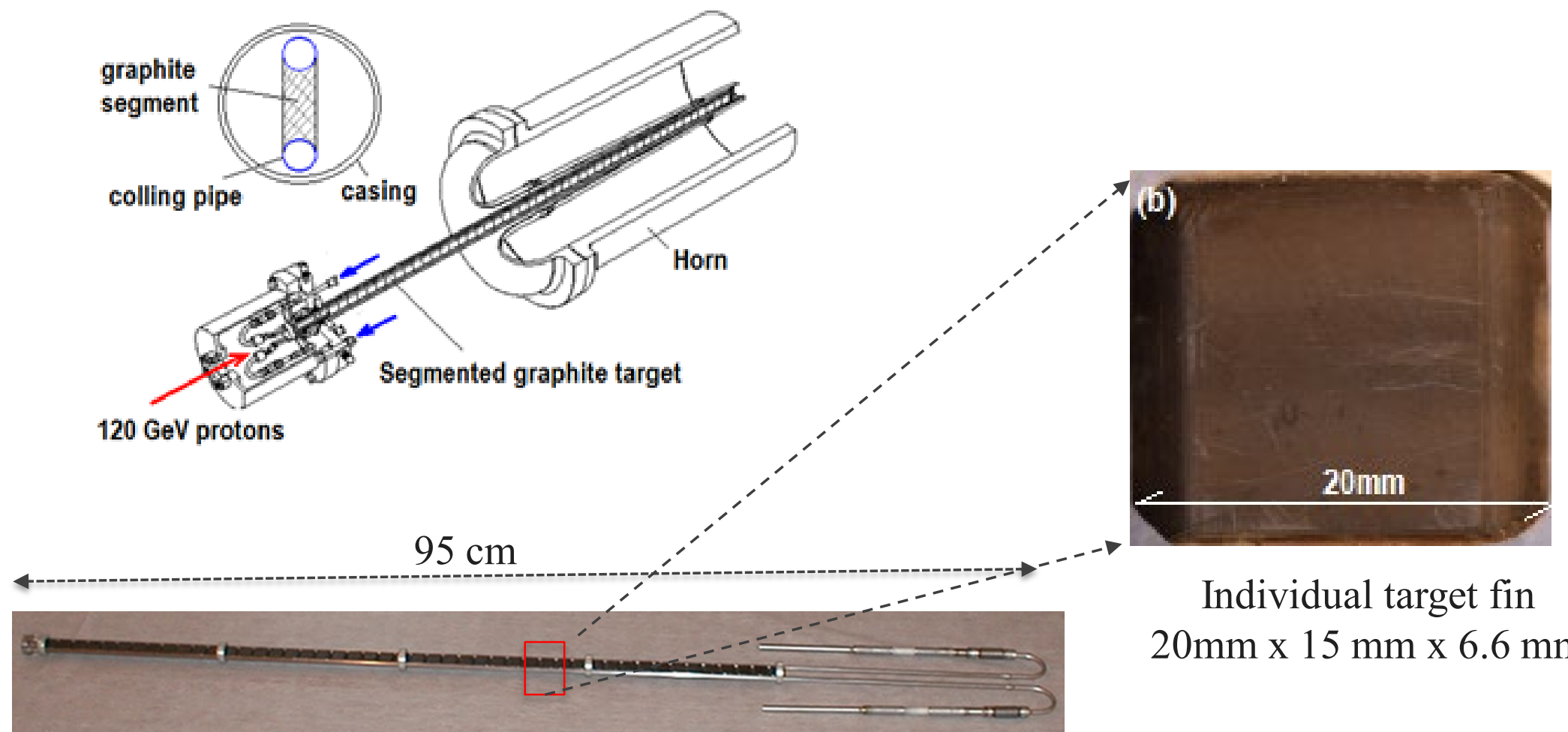

Segmented Fins- 47 pieces

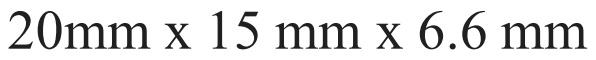

Material : POCO ZXF 5Q graphite

Isotropic 


\section{Background- Fracture fin}

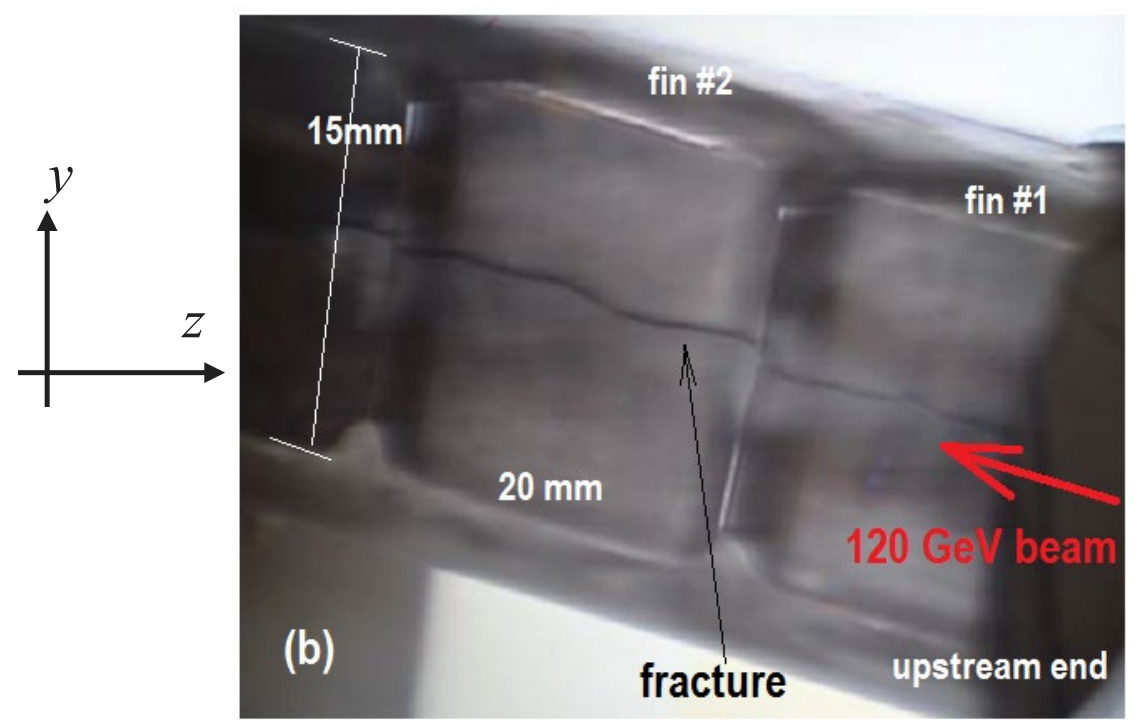

Beam Parameters:

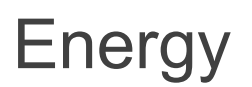

$: 120 \mathrm{GeV}$

Beam sigma : $1.1 \mathrm{~mm}$

Spill duration : $10 \mu \mathrm{sec}, 4 \times 10^{13}$ protons/pulse

Duty cycle $\quad: 1.87 \mathrm{sec}$

Peak fluence : $8.6 \times 10^{21}$ protons $/ \mathrm{cm}^{2}$

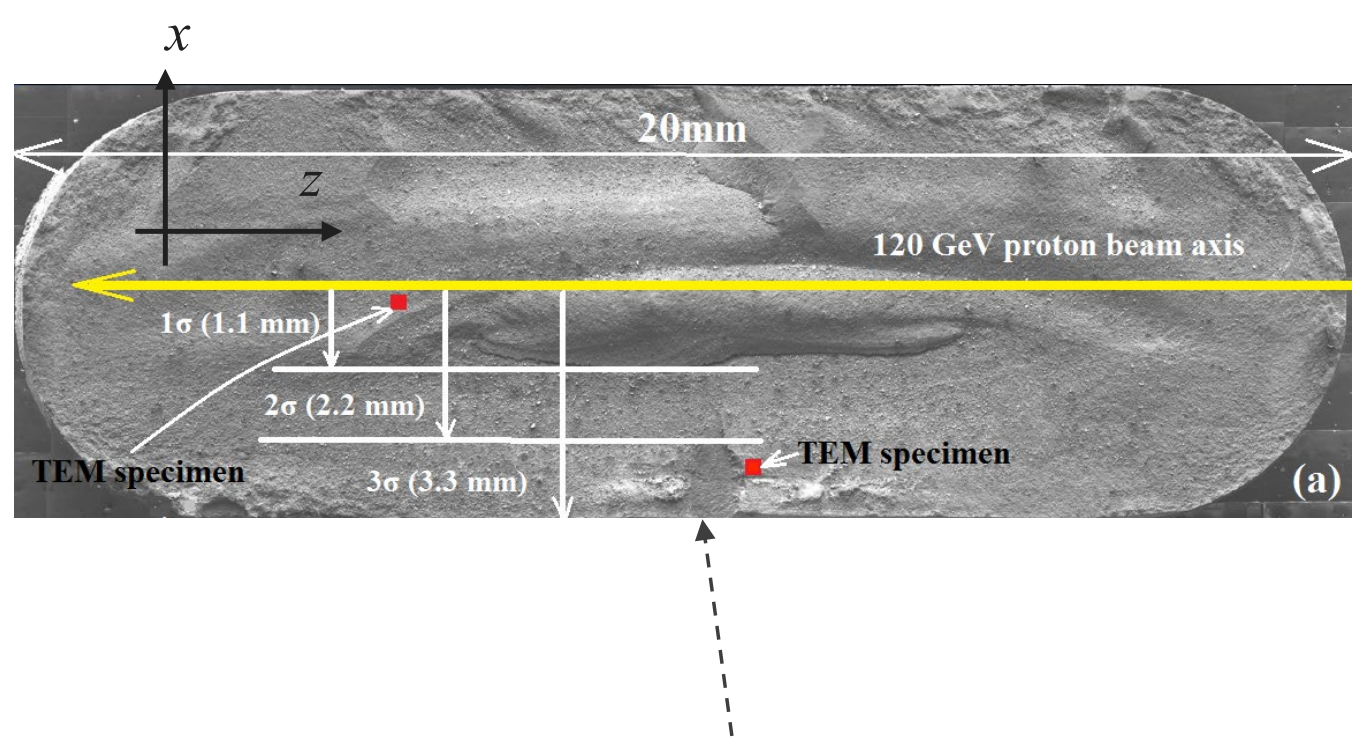

Bulk swelling of $\sim 2 \%$ 


\section{POCO ZXF 5Q Graphite microstructure}
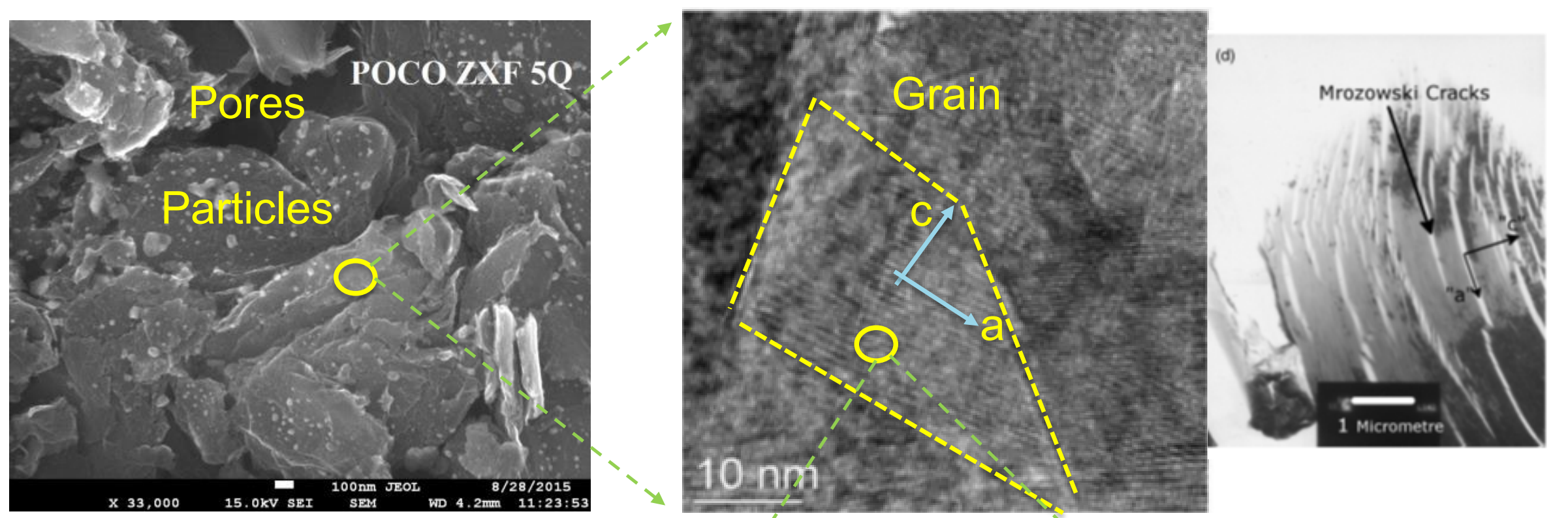

- Less Mrozowski cracks

- Randomly oriented grains

- isotropic

- Pores \& particles microns

- Particles packed with many

- Fine grains ( 30nm)

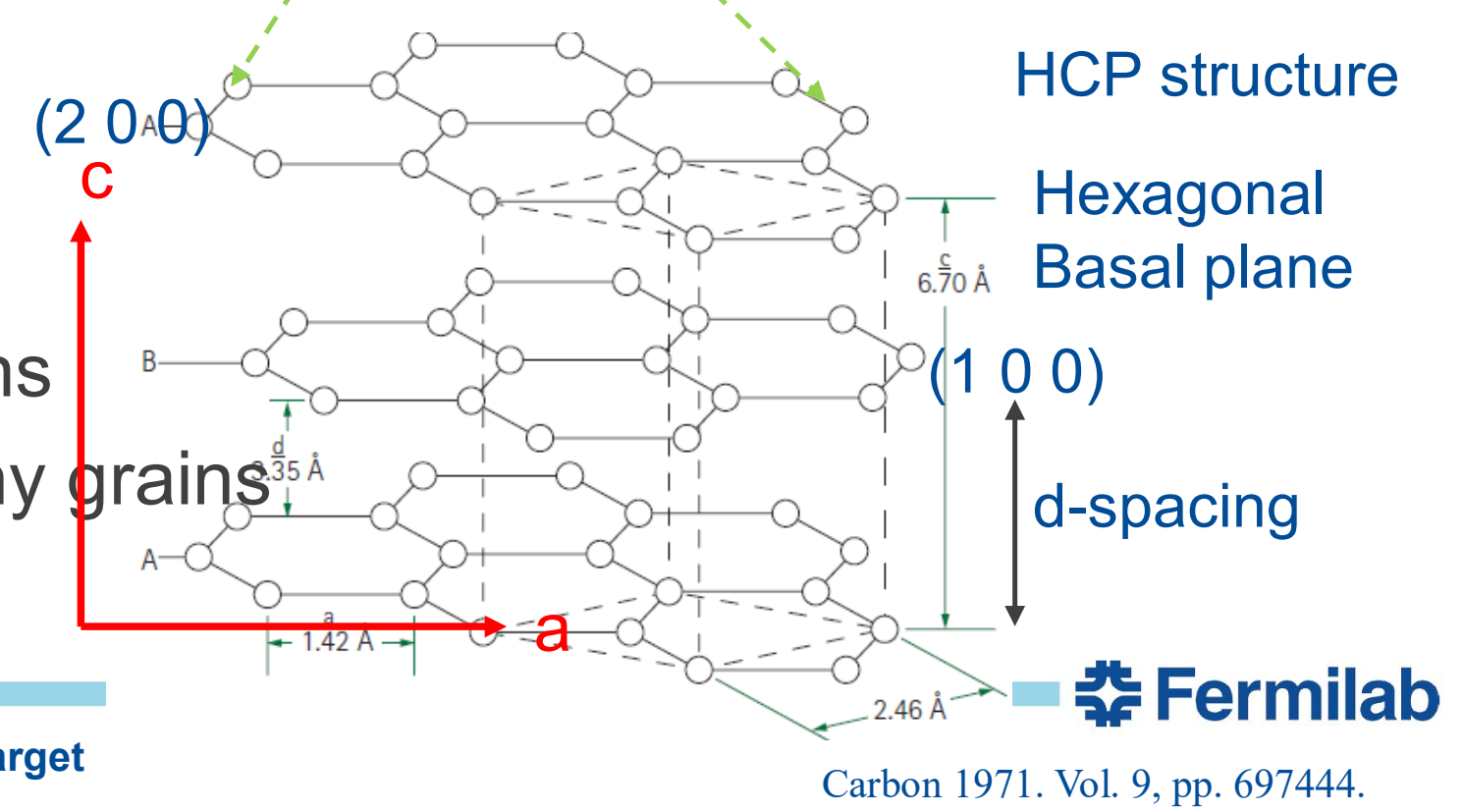




\section{Graphite Swelling $\rightarrow$ Fluence factor}

$\underline{\text { XRD - Pyrolytic carbon }}$

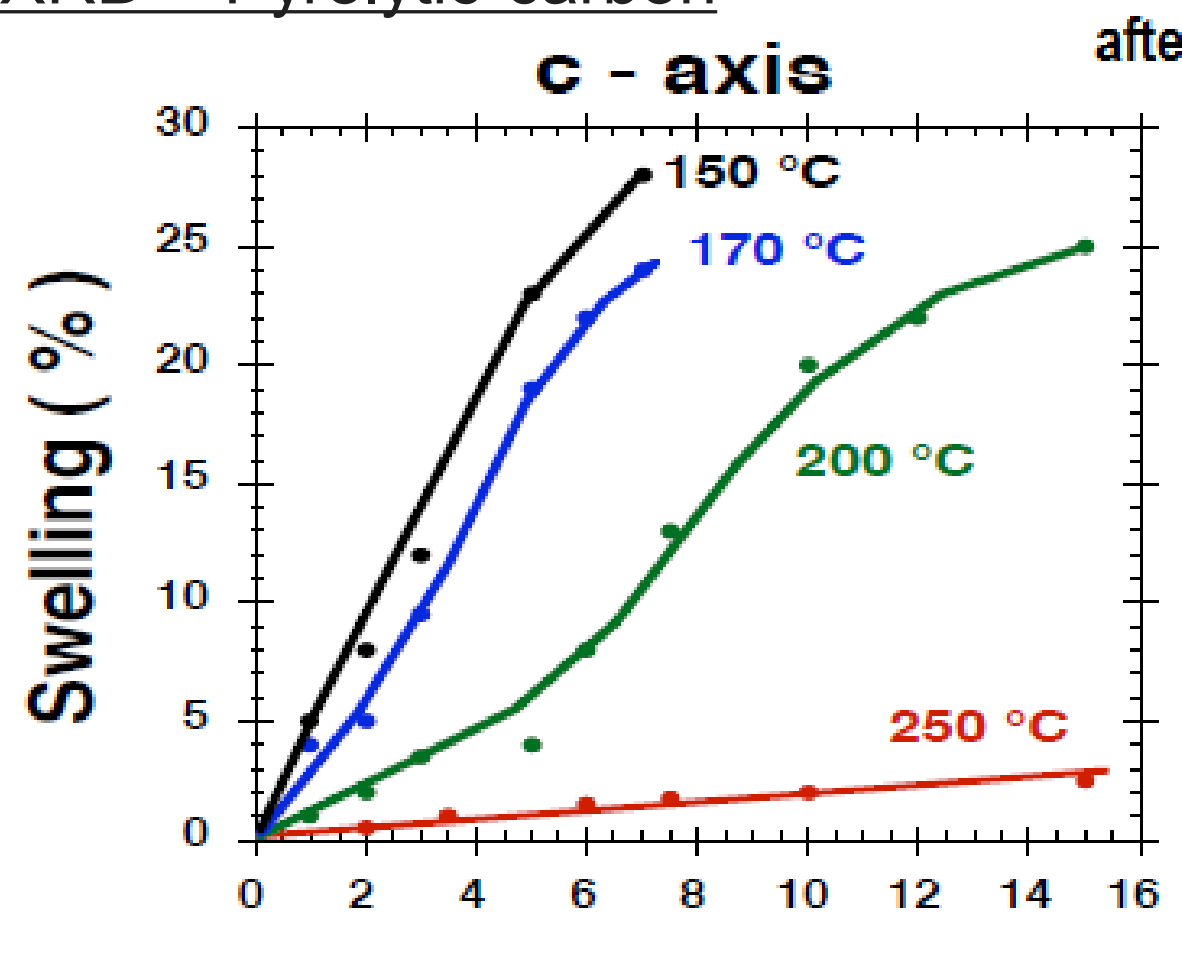

after Kelly (1986)

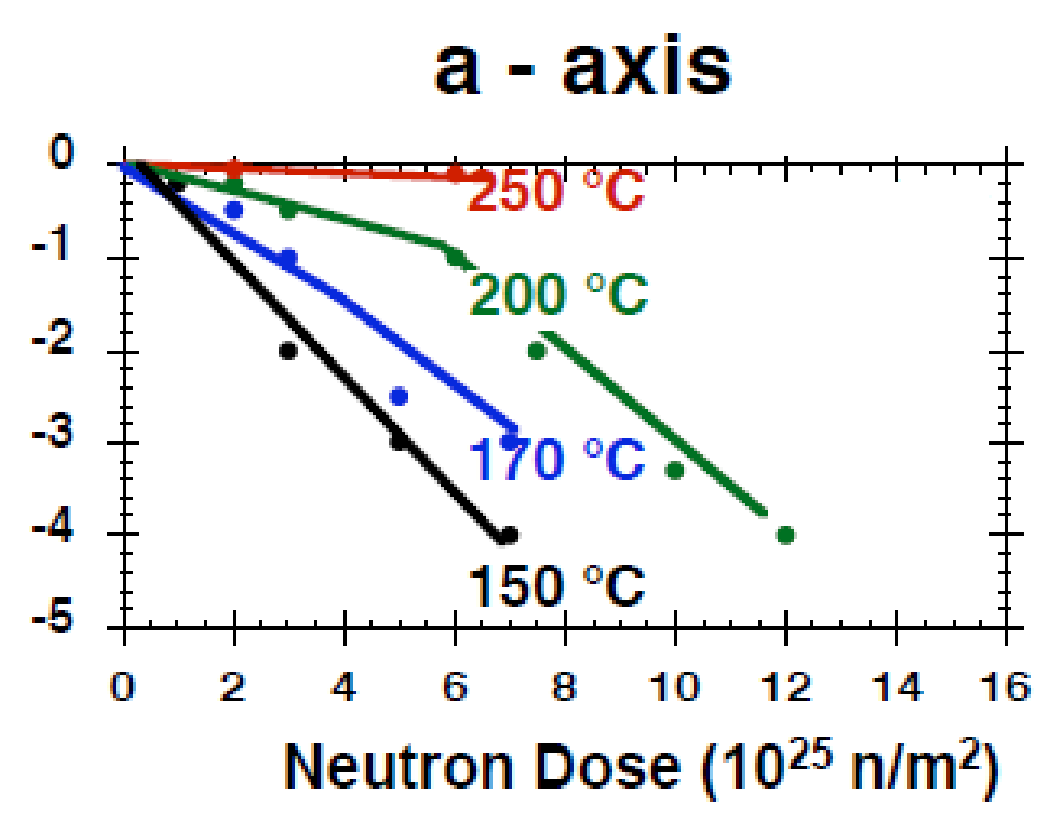

Neutron Dose $\left(10^{25} \mathrm{n} / \mathrm{m}^{2}\right)$

$\mathrm{C}$-axis expansion is more than a-axis contraction

Swelling $\rightarrow$ f(fluence, temperature)

- More swelling at lower temperature 


\section{Graphite Swelling $\rightarrow$ XRD- POCO ZXF5Q}
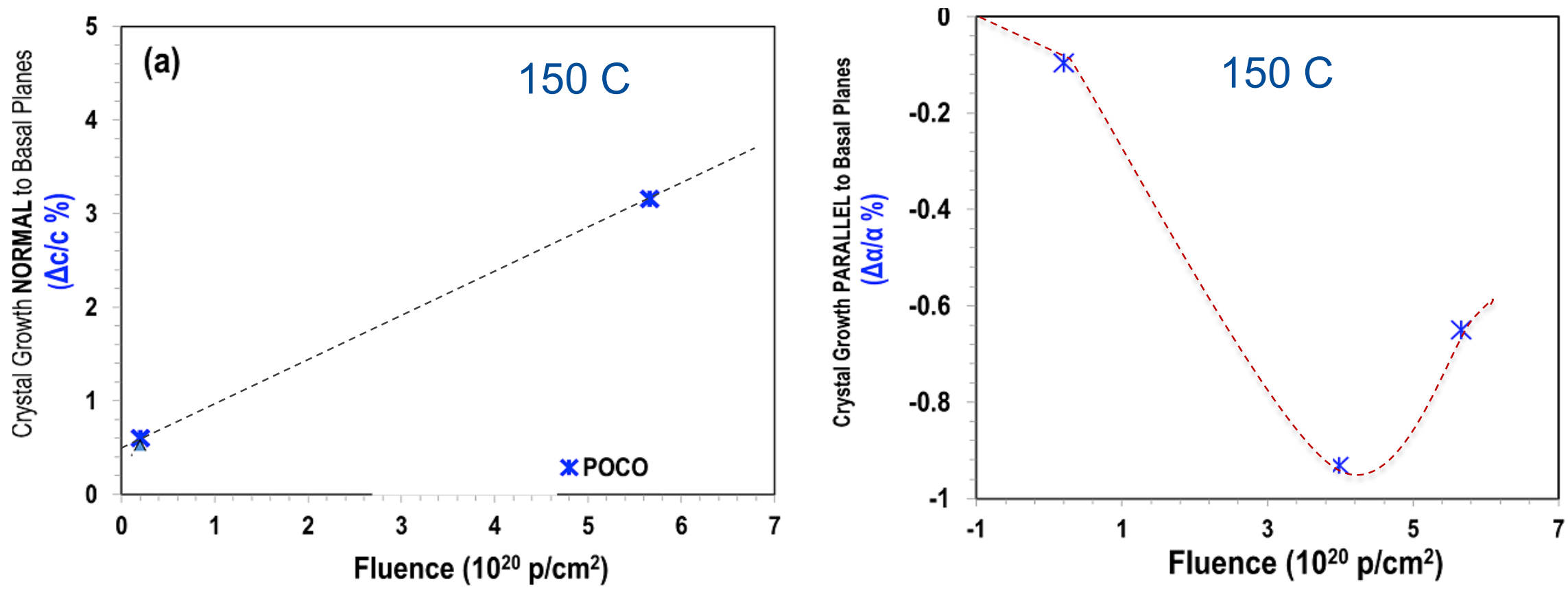

$\mathrm{C}$-axis expansion is more than a-axis contraction $\rightarrow$ net expansion
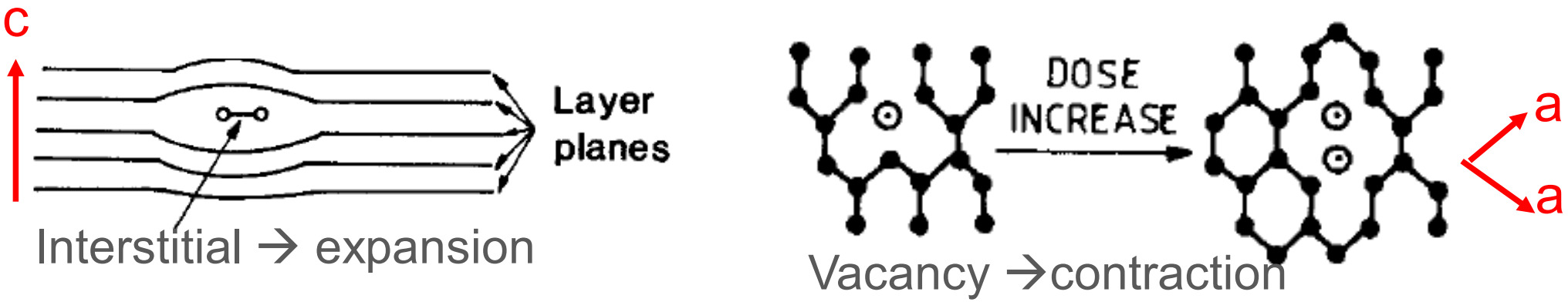


\section{Swelling factor function}

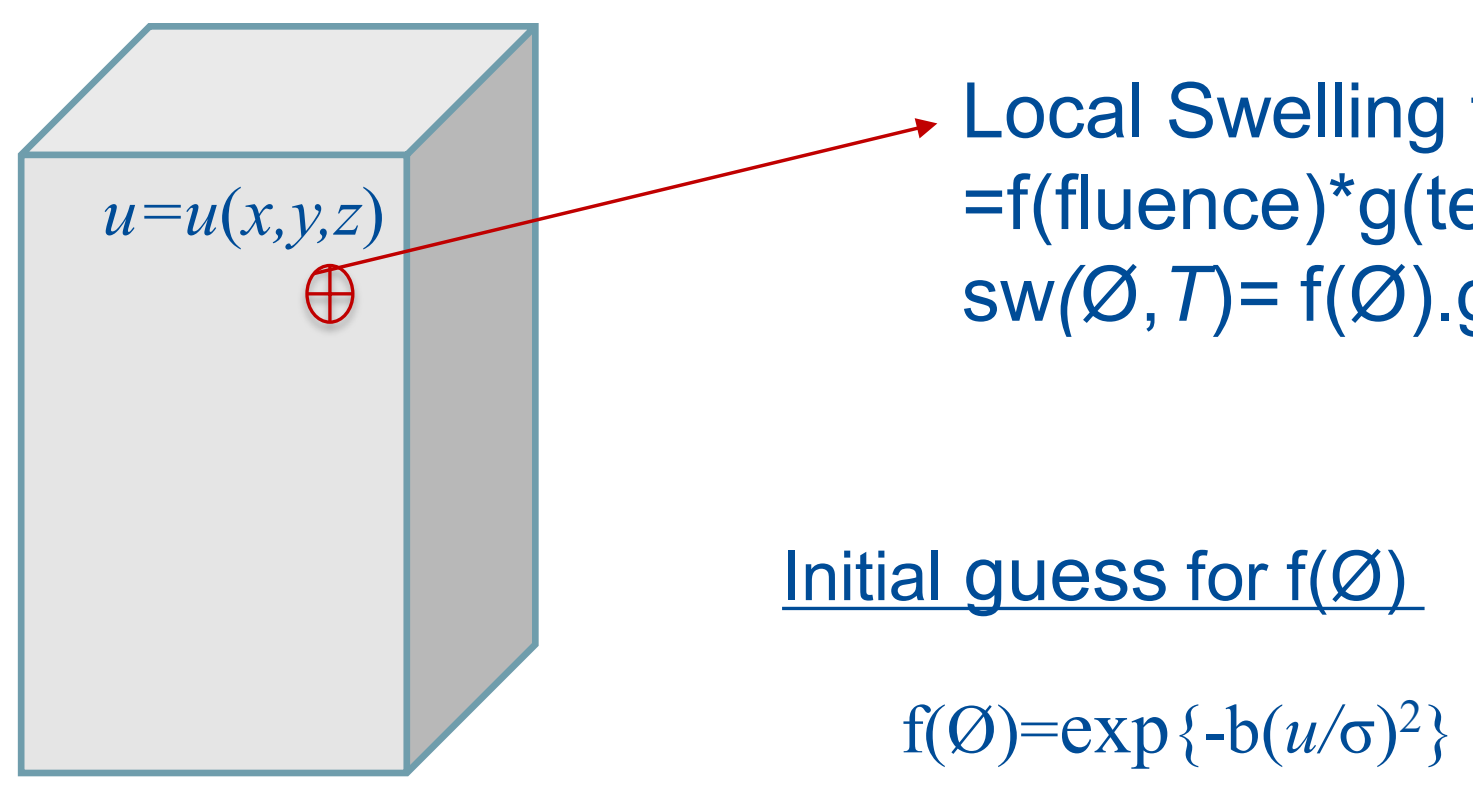




\section{Swelling factor function}

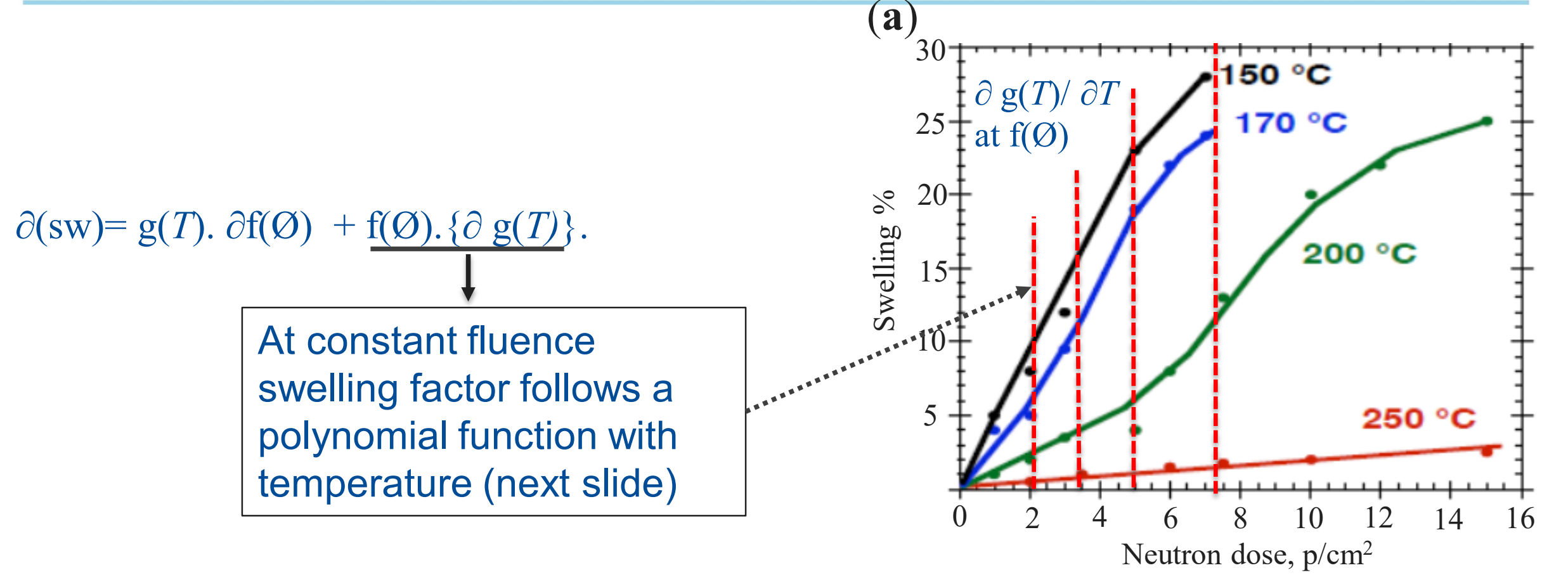

Attempting to find form of the function from the swelling trend 


\section{Swelling $\rightarrow$ Temperature Factor}
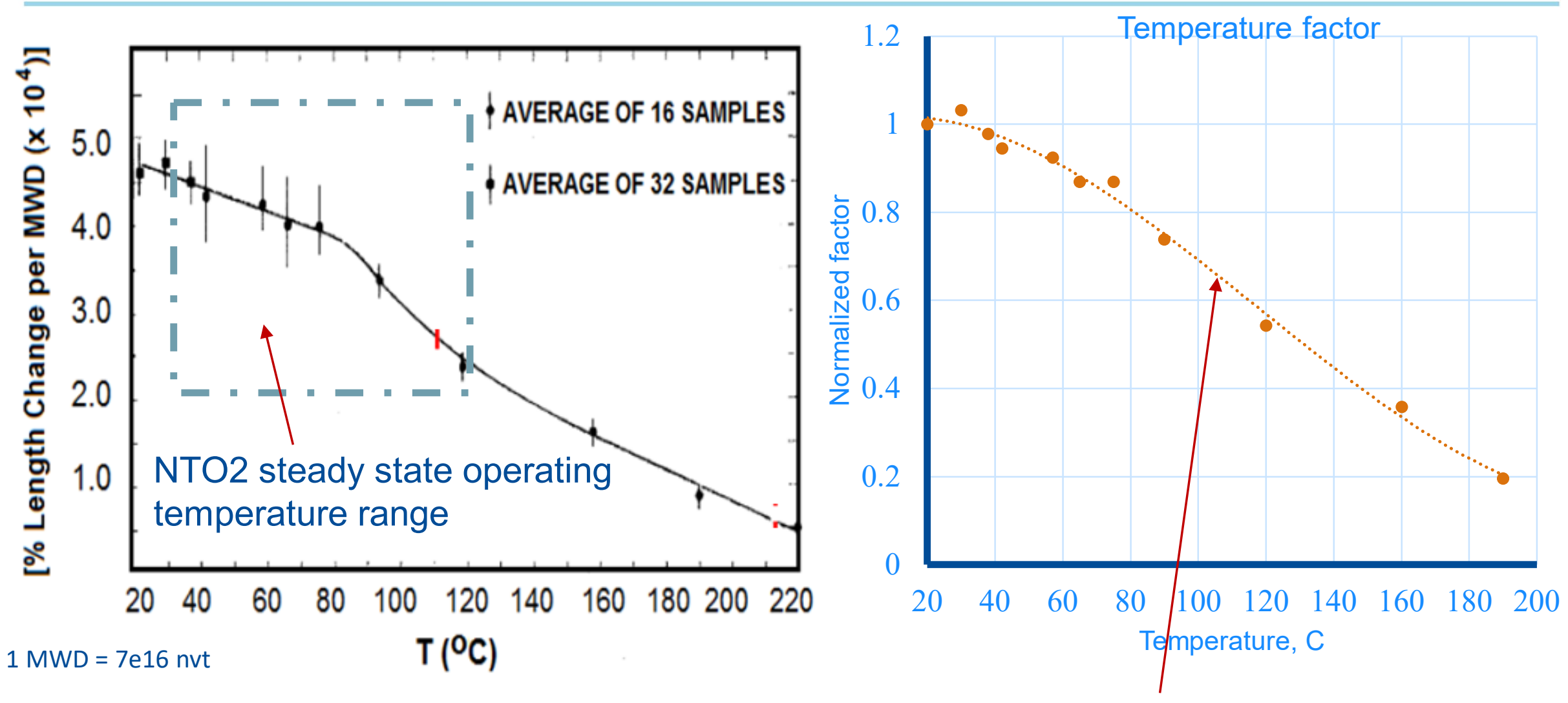

$\partial \mathrm{g}(T)=2 \times 10^{-7} T^{3}-7 \times 10^{-5} T^{2}+1.5 \times 10^{-3} T+1.0083$

Pronounced swelling at lower temperatures 


\section{Steady State Thermal Analysis}

\section{$1 / 8^{\text {th }}$ symmetry model}

(a)

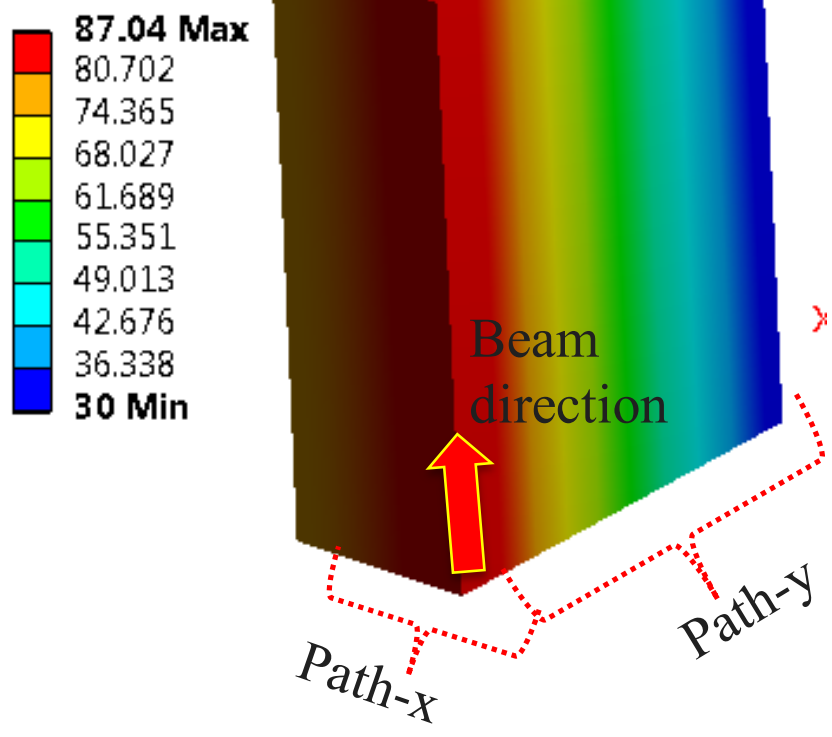

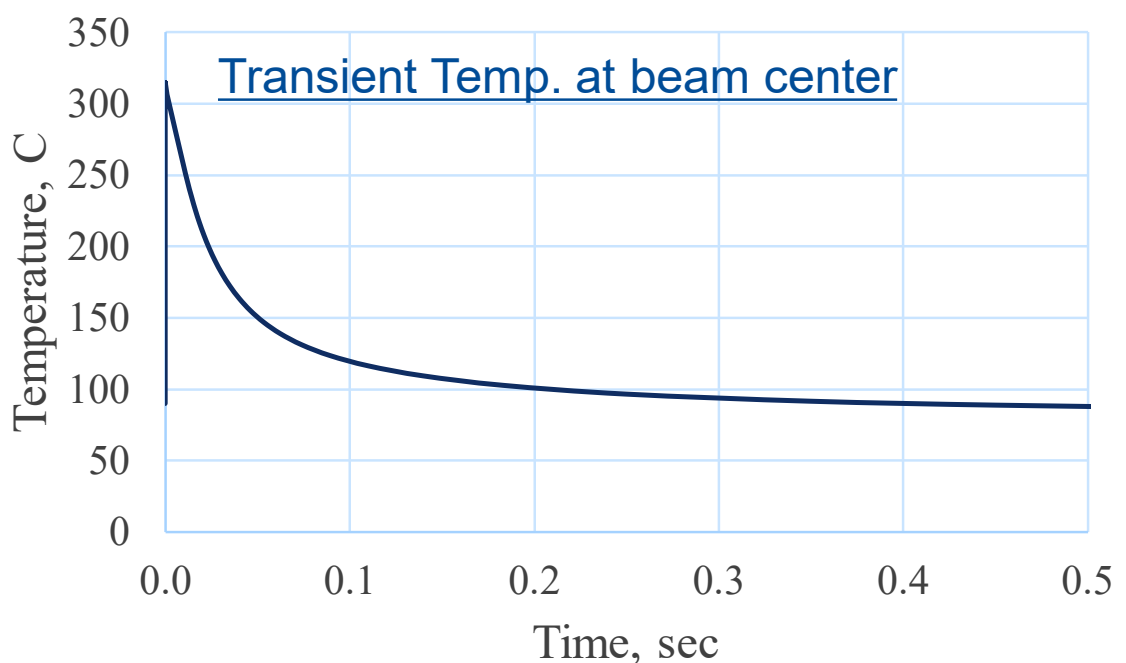

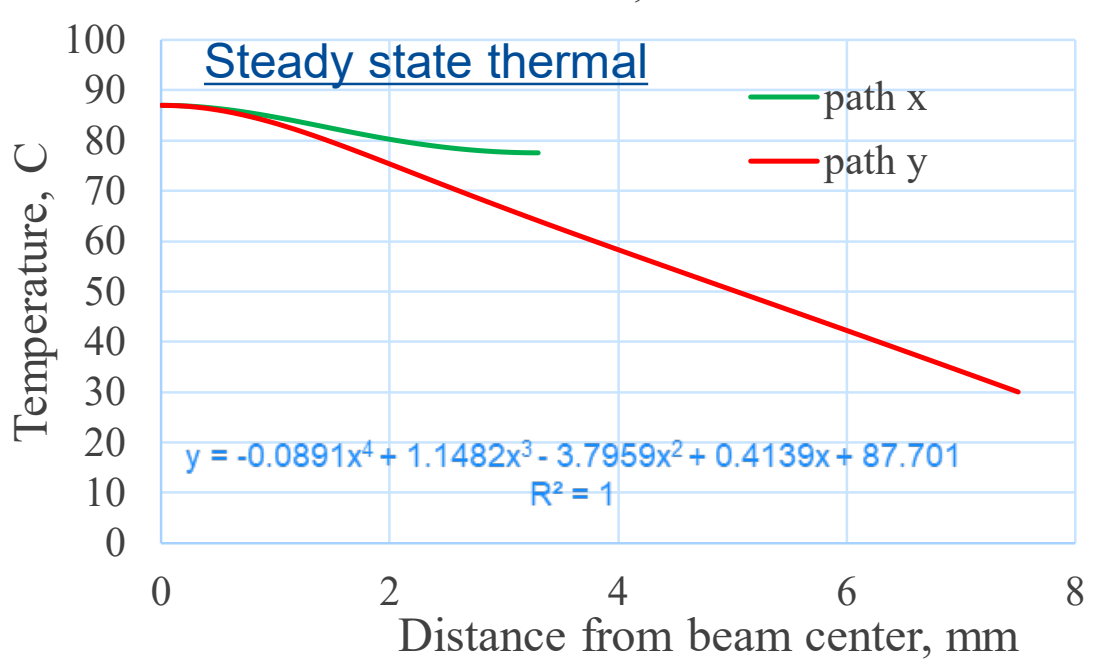

Average irradiation temperature $30 \mathrm{C} \sim 87 \mathrm{C}$ 


$$
\operatorname{sw}(u, T)=C \cdot \exp \left\{-b\left(\frac{u}{\sigma}\right)^{2}\right\} \cdot\left\{5 \times 10^{-8} T^{4}-2 \times 10^{-5} T^{3}+5 \times 10^{-4} T^{2}+1.0083 T+1\right\}
$$

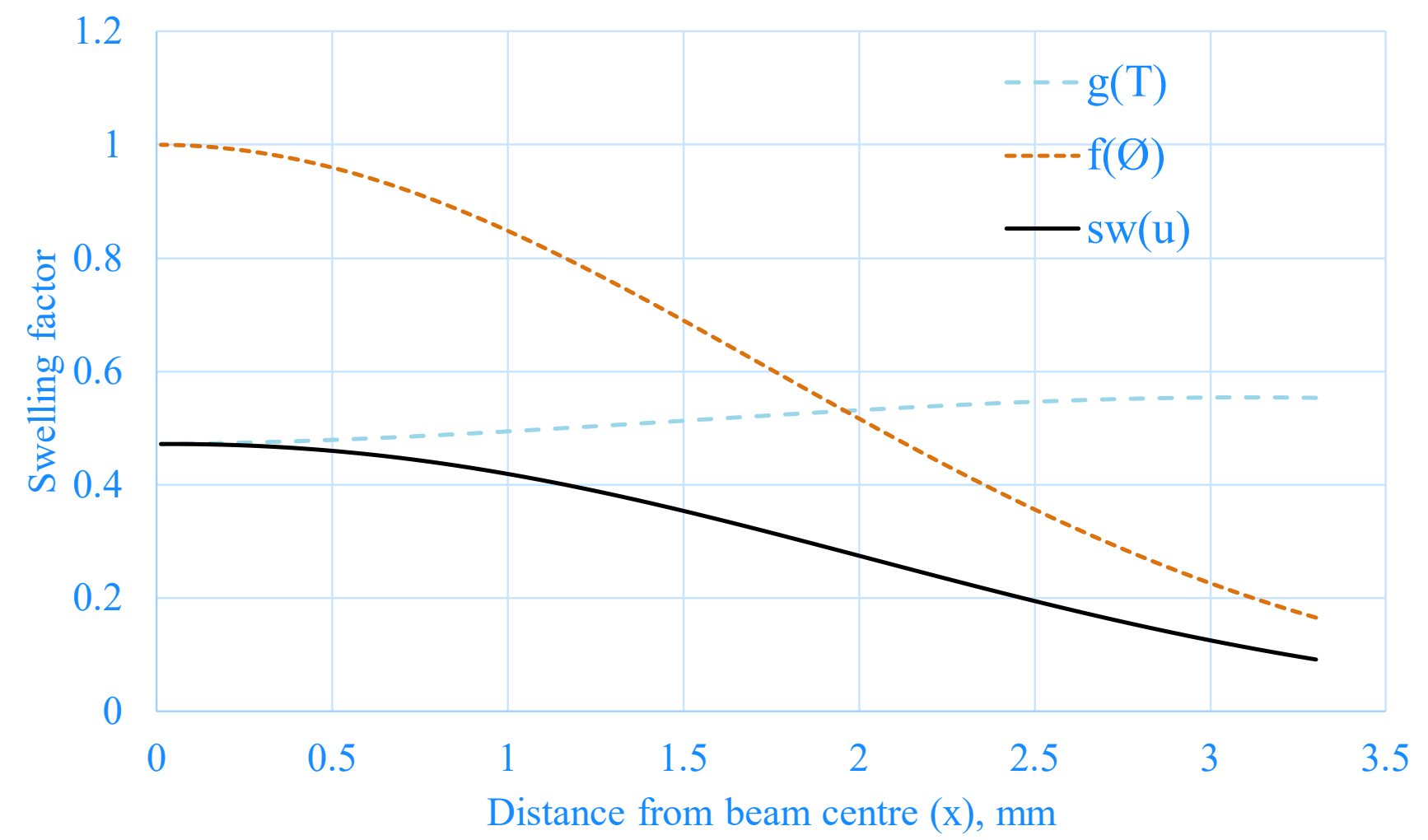

Swelling is reduced at center due to relatively high irradiation temperature 


\section{XRD data NTO2}

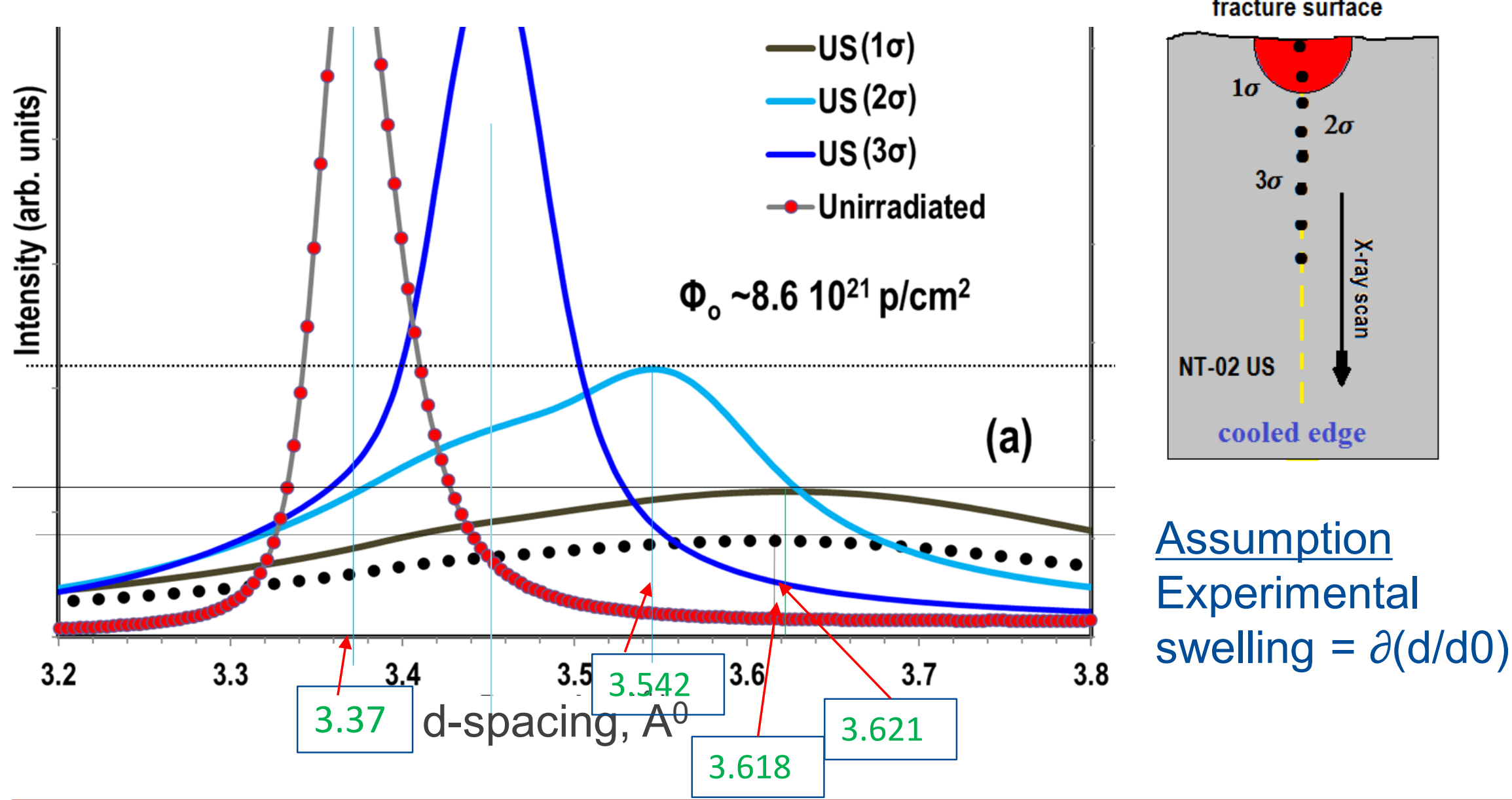

XRD scan spot size is $50 \mu \mathrm{m} \times 50 \mu \mathrm{m} \times 10 \mathrm{~mm}$

In FEA, element size is made $\sim 50 \mu \mathrm{m}$ so that it would match to XRD scan volume 


\section{Determining Swelling Coefficient}

\begin{tabular}{|c|c|c|c|c|c|}
\hline $\mathrm{U}(\mathrm{mm})$ & $\begin{array}{c}\text { Temper } \\
\text { ature }\end{array}$ & sw & $\begin{array}{c}\mathrm{d}- \\
\text { spacing }\end{array}$ & $\partial(d / d 0)$ & C \\
\hline 0 & 88 & 0.779 & 3.621 & 0.074481 & 0.000894 \\
\hline 1.1 & 84 & 0.686 & 3.618 & 0.073591 & 0.001073 \\
\hline 2.2 & 81 & 0.442 & 3.542 & 0.051039 & 0.001211 \\
\hline 3.3 & 78 & 0.210 & 3.45 & 0.023739 & 0.001245 \\
\hline
\end{tabular}

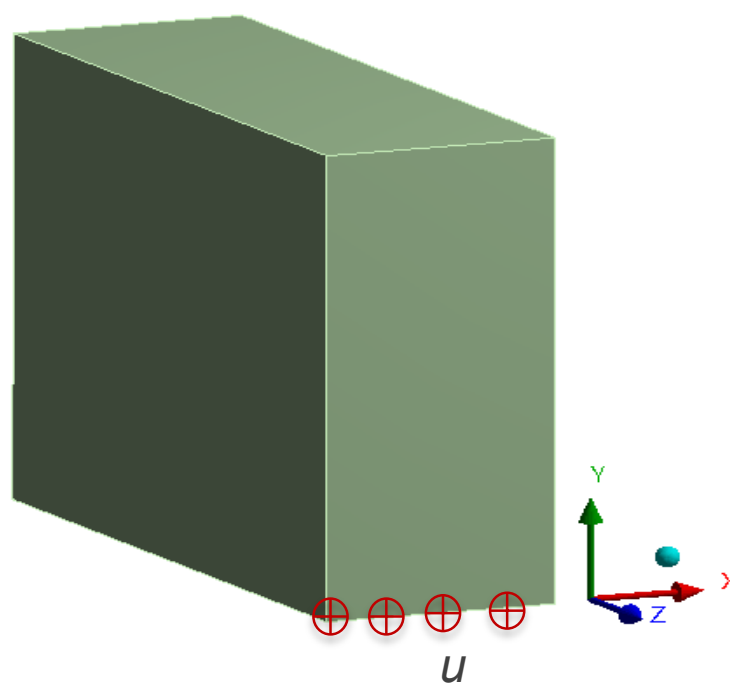

C. $\exp \left\{-b\left(\frac{u}{\sigma}\right)^{2}\right\} \cdot\left\{5 \times 10^{-8} T^{4}-2 \times 10^{-5} T^{3}+5 \times 10^{-4} T^{2}+1.0083 T+\right.$

Multi-variate regression analysis:

Varying "b" and " $\sigma$ "to get $C$ curve as flat as possible

$\rightarrow \mathrm{b}=0.155, \mathrm{C}=0.00107, \sigma=1.11$

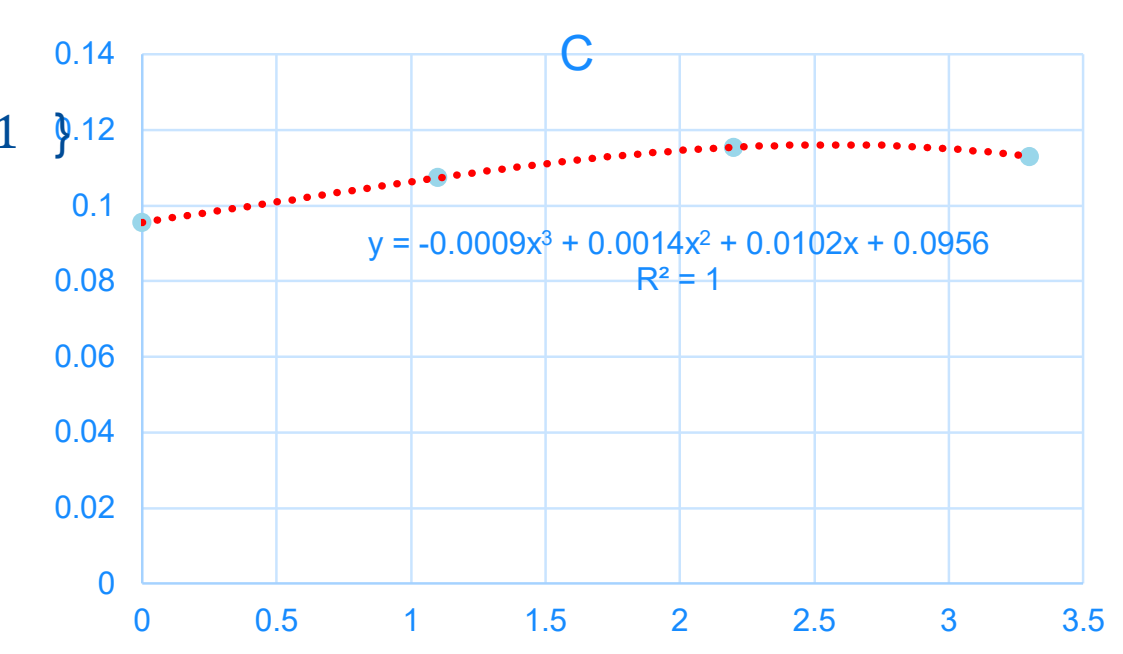

$$
\begin{aligned}
& \operatorname{sw}(u, T) \\
& =0.0011 . \exp \left\{-0.155\left(\frac{u}{1.11}\right)^{2}\right\} \cdot\left\{5 \times 10^{-8} T^{4}-2 \times 10^{-5} T^{3}+5 \times 10^{-4} T^{2}+1.0083 T+1\right\}
\end{aligned}
$$

14 Irradiation Induced Failure analysis NUMI Target 


\section{Young's Modulus variation with Fluence $\varnothing$}
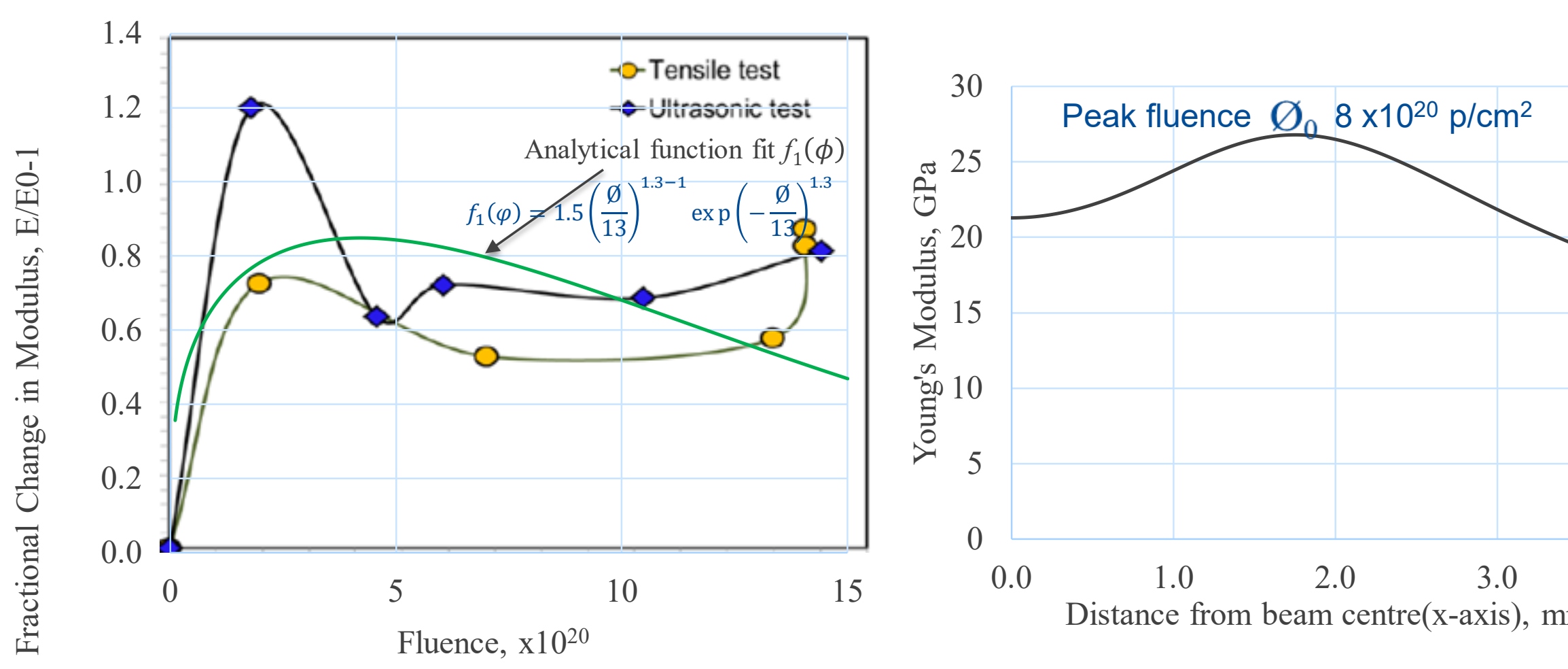

"E" varies with $\varnothing$ $\varnothing$ varies with location. $\varnothing=\varnothing_{0} \exp \left\{-0.5(u / 1.1)^{2}\right\}$ This two relationship is used to map is use

$$
\mathrm{E}=\mathrm{E} 0\left[1+f_{l}(\varnothing)\right]
$$




\section{Model- Geometry}

$1 / 8^{\text {th }}$ Model $\rightarrow$ Symmetric in $\mathrm{x}, \mathrm{y}$, and $\mathrm{z}$ direction

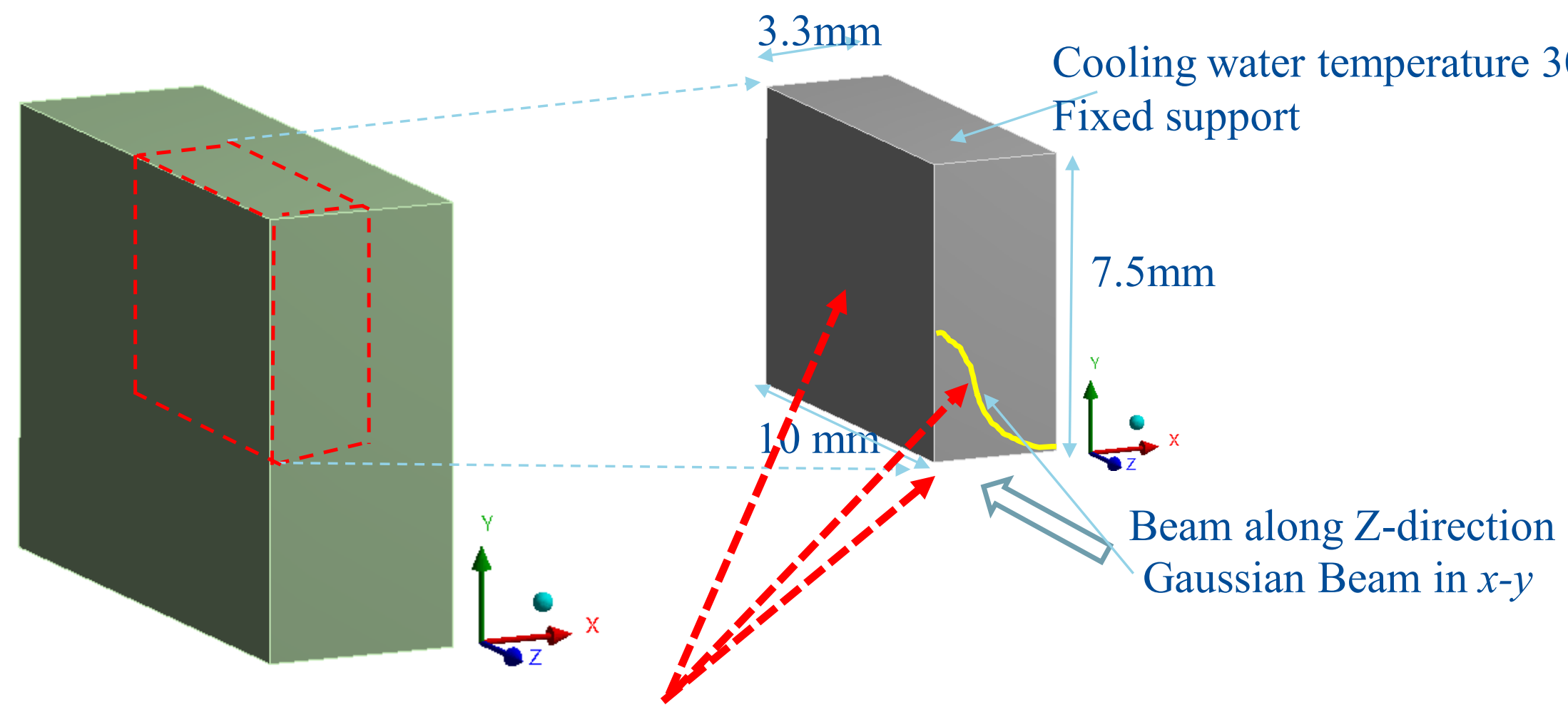

Symmetric Boundary Condition on $\mathrm{x}, \mathrm{y}$ and $\mathrm{z}$ plane

$$
\begin{aligned}
u_{i, i} & =0 \\
\theta_{i, j} & =0 \quad i \neq j \\
& =\text { free } i=j
\end{aligned}
$$




\section{FE Model}

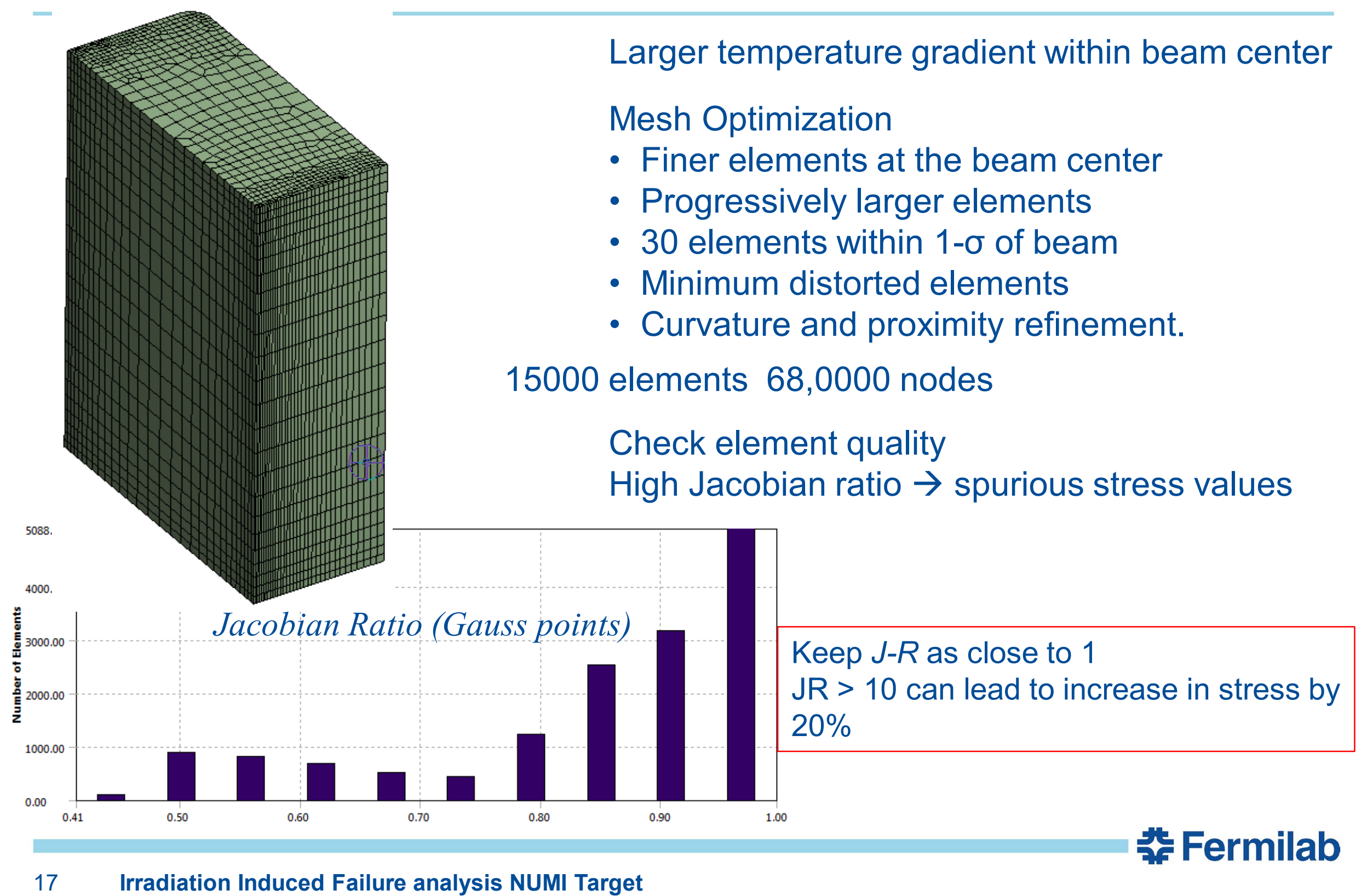




\section{FEM Implementation of Empirical Formula}

Total Strain

$$
\varepsilon=\dot{\varepsilon}_{e l}+\dot{\varepsilon}_{p l}+\dot{\varepsilon}_{c r}+\dot{\varepsilon}_{t h}
$$

Linear swelling model $\quad \dot{\varepsilon}_{s w}=C \dot{\Phi}_{t}$

Swelling Strain $\dot{\varepsilon}_{s w}=\varepsilon_{S w}\left(t, T, \Phi_{t}, \sigma\right)$

$\times x$ Surtace body

由... $\times$ Solid

冒 Commands (APDL)

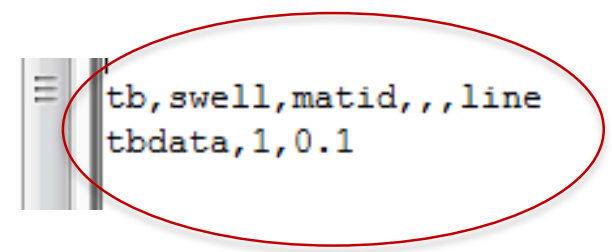

$\left\{\varepsilon^{s w}\right\}=\left\lfloor\begin{array}{llllll}\varepsilon_{\mathrm{n}}^{\mathrm{sw}} & \varepsilon_{\mathrm{n}}^{\mathrm{sw}} & \varepsilon_{\mathrm{n}}^{\mathrm{sw}} & 0 & 0 & 0\end{array}\right]^{\top} \Rightarrow$

No shear, only volume change

Normal Stress 2

19. Maximum Principal Elastic St

$\checkmark$ is User User Defined Result

Giser User Defined Result 2

Transient (F5)

㼛远 Initial Conditions

Wi. Analysis Settings

冝: swelling

冒: modulus

冝; swelling 2

글 Imported Load (E6)

II, Remote Displacement

Solution (F6)

I] Solution Information

Equivalent Stress

19 Normal Stress 4

Normal Stress 3

$\forall$ Giser User Defined Result

Normal Stress

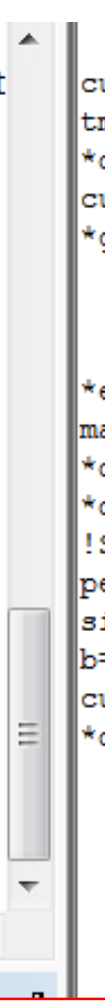

currnode $1=0$

$t \max =0$

*do, i, 1, numnodes

currnode1=ndnext (currnode1)

* get, tem, node, currnode1, ntemp

*if, tem, gt, tmax, then

tmax $=$ tem

*endiffPDL Scripts

max_temp $=$ tmax

*dim, locax, array, numnodes

*dim, flu value, array, numnodes

! Swelling only factor

peak $=0.107$

sigma $=1.11$

$\mathrm{b}=0.155$

currnode $=0$

*do, i, 1, numnodes

currnode=ndnext (currnode)

1_ $\mathrm{x}=\mathrm{nx}$ (currnode)

1_y $=$ ny (currnode)

flu_fact $=\exp \left(-b^{*}\left(1 \_x^{* * 2+1} \mathrm{y}^{* *}\right.\right.$ $\operatorname{locax}(i)=1 \mathrm{x}$

flu_value $(\bar{i})=f l u \_f a c t$

\section{由}

Normal Stress 2

Maximum Principal Elastic St

$\checkmark$ Giser User Defined Result

$\checkmark$ Gise User Defined Result 2

\section{Transient (F5)}

住运 Initial Conditions

IN. Analysis Settings

盾: swelling

$\frac{\text { ri modulus }}{\text { 集: swelling 2 }}$

当 Imported Load (E6)

III Remote Displacement

\section{Solution (F6)}

1.] Solution Information

Equivalent Stress

10 Normal Stress 4

10 Normal Stress 3

Gị User Defined Result

Normal Stress

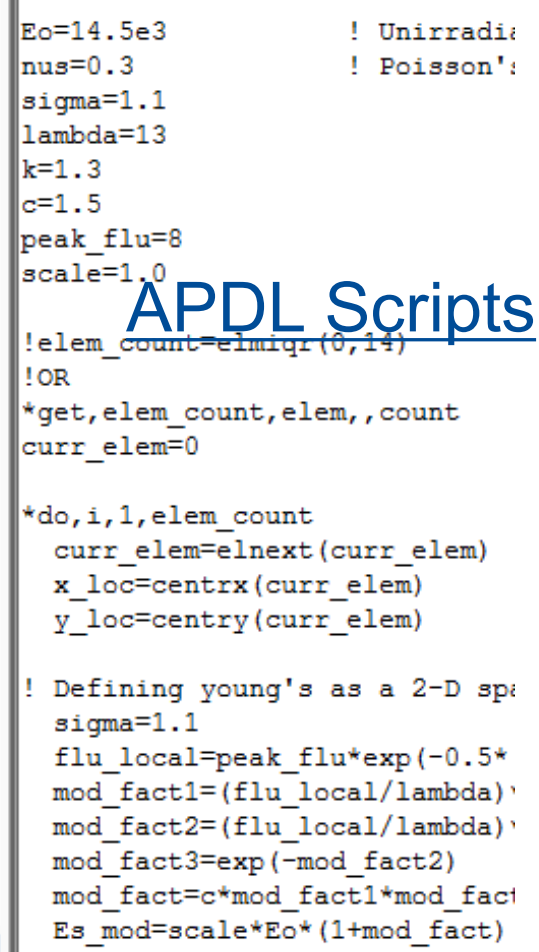
$\mathrm{mn}$ FY ; $\mathrm{F}=\mathrm{mna}$ 


\section{Simulation Frame-work}

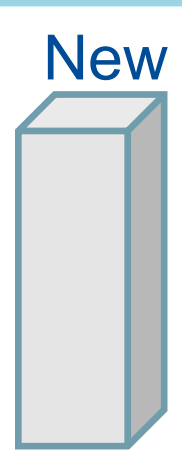

After years of operation

One beam pulse

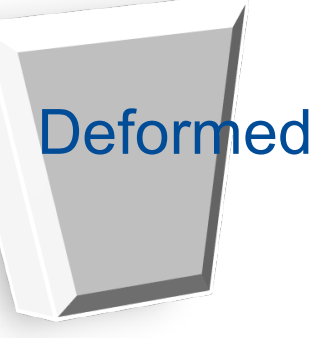

Steady State Thermal

Analysis

Static structural analysis

- swelling factor as function of fluence,temperature $\operatorname{sw}(\varnothing, T)$

- Young's modulus=f(fluence)

- CTE $\rightarrow f($ Temperature)

- Solve stress, strain , deformation states

Few micro-secs of high temperature is not enough to influence any swelling (annealing)

Transient Thermal-Dynamic analysis

- Initialize domain with $\sigma, \varepsilon, u$ from static structural

- Turn off $g(T)$ portion of $\operatorname{sw}(\varnothing, T)$

- Young's modulus=f(fluence)

- CTE $\rightarrow f($ Temperature) 


\section{Static Structural analysis $-\sigma_{\mathrm{yy}}$ Distribution}
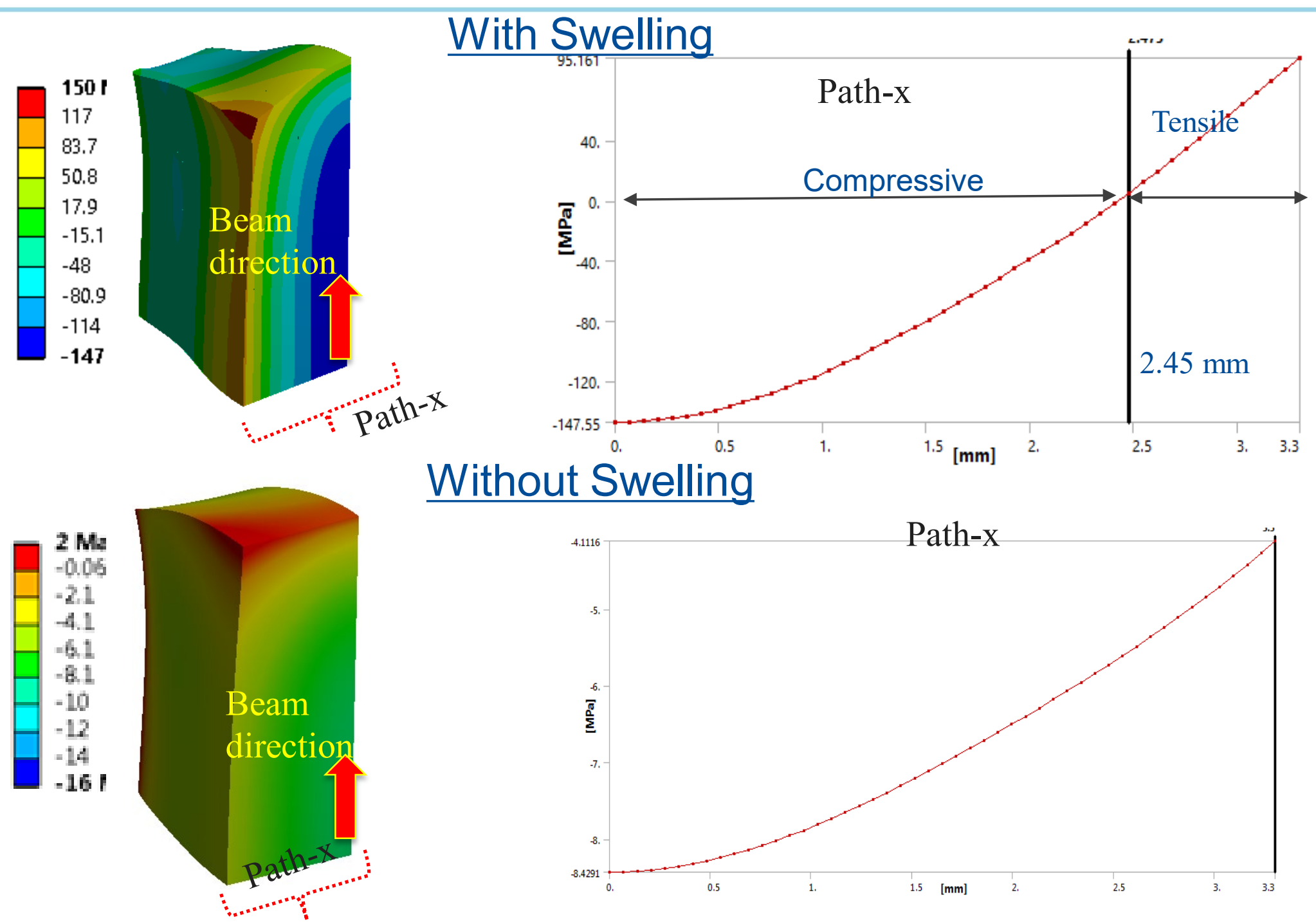

Without Swelling

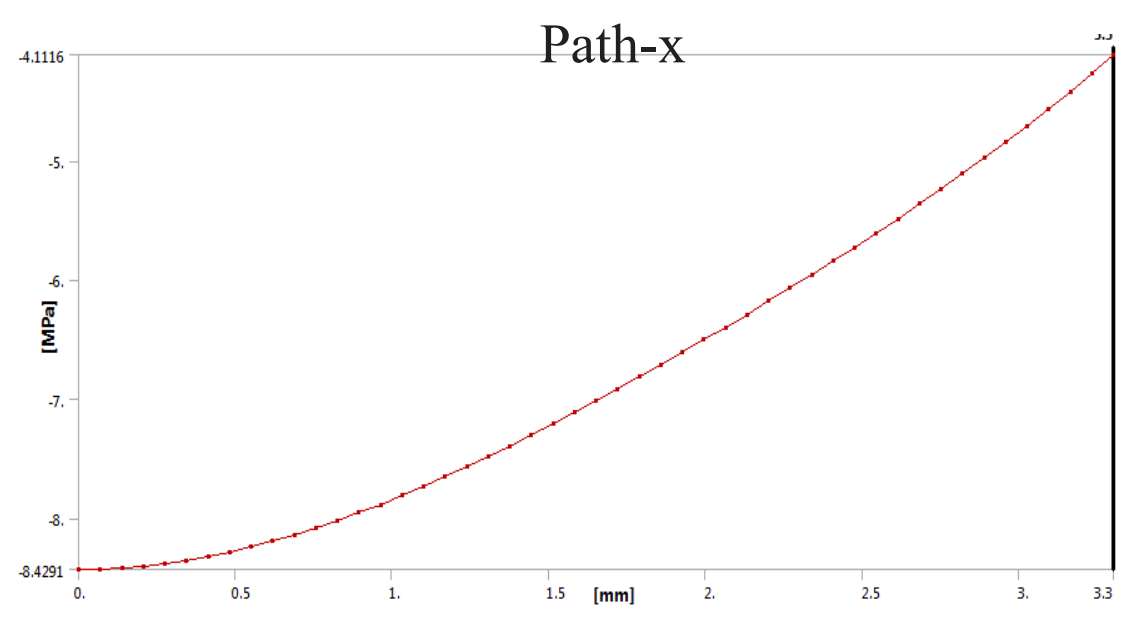

State just before beam pulse after number of years of exposure to neutrons 


\section{Simulation validation- failure strength}

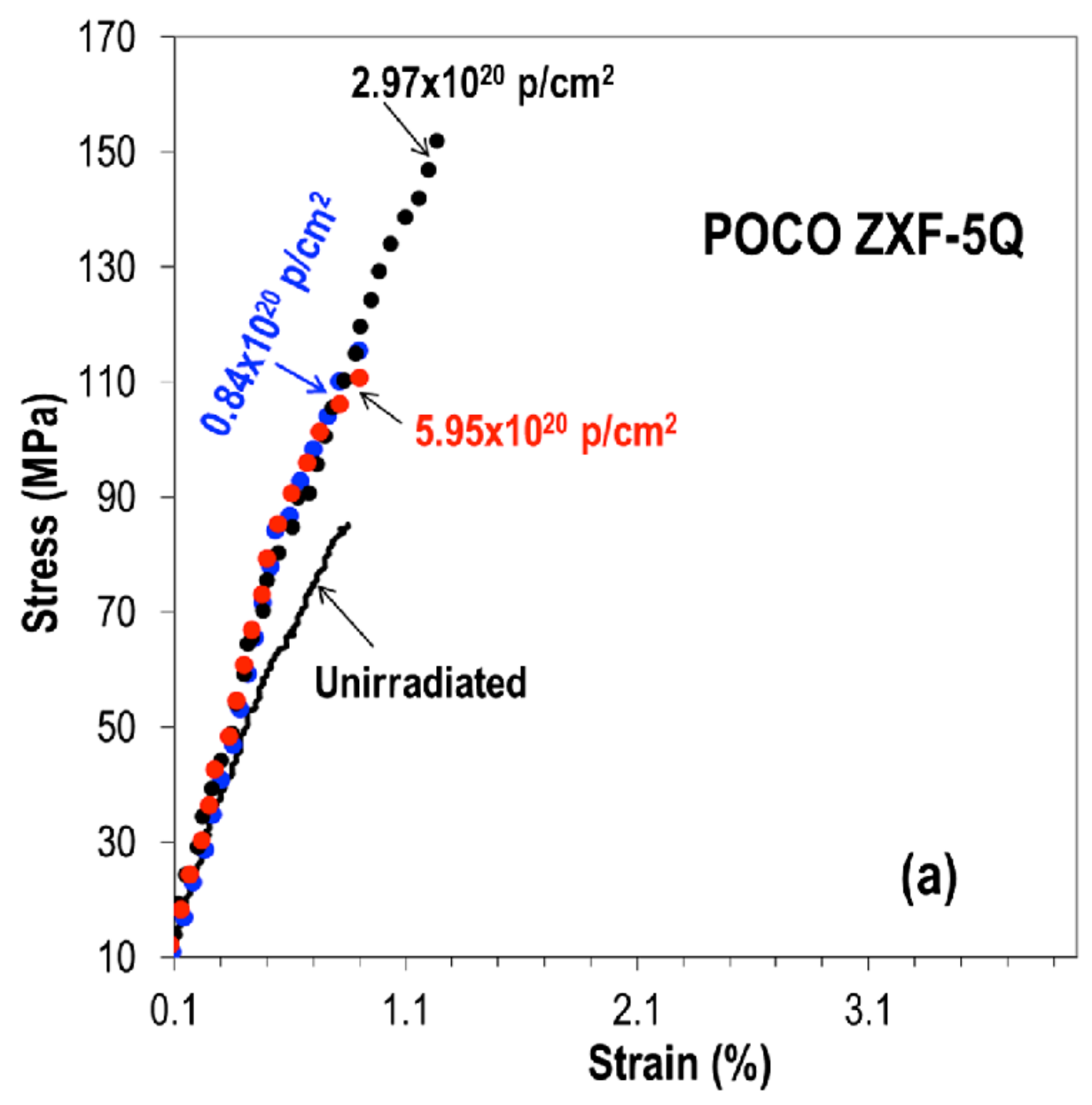

Swelling formulation raised the stress level up to $150 \mathrm{MPa}$ 


\section{Simulation Validation- amount of swelling}

\section{Deformed location}

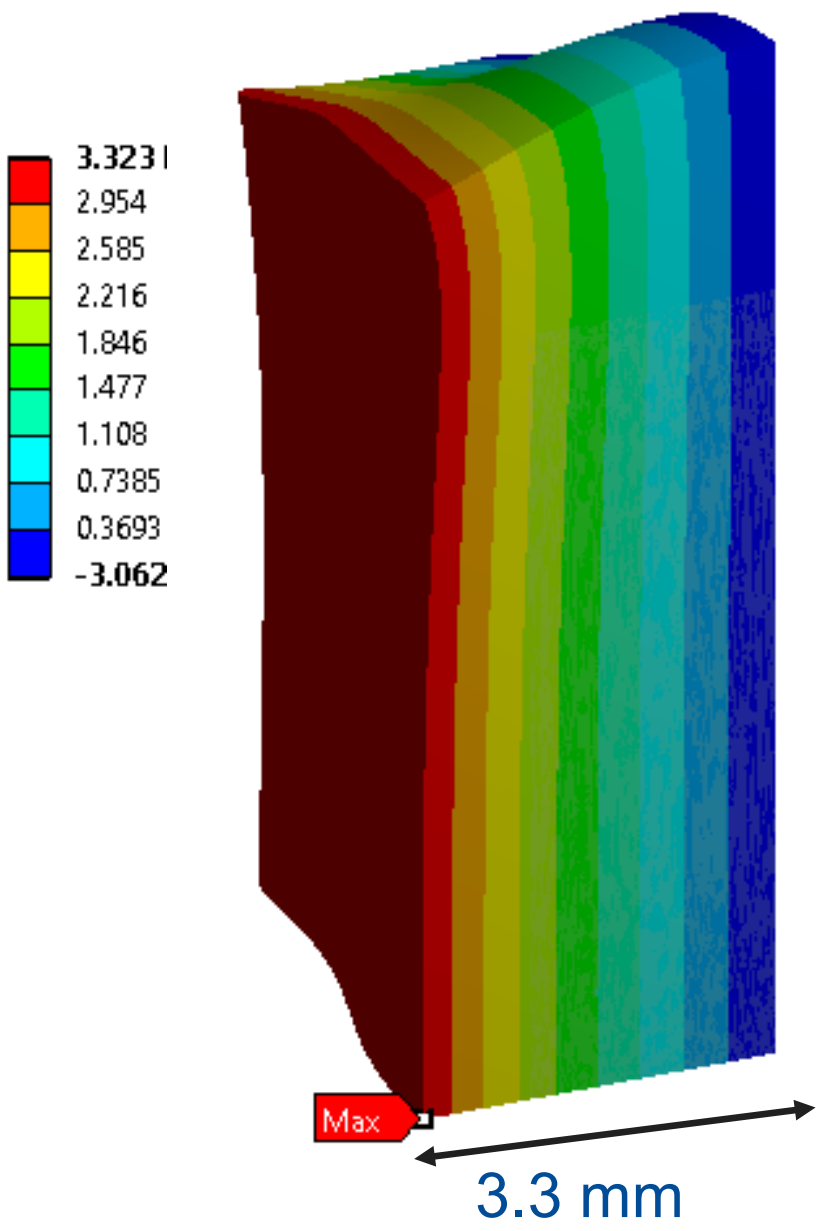

From Simulation
$(3.323-3.3)^{*} 100 / 3.3=0.7 \%$

Observed bulk swelling at that location 2\%

Difference of factor of 2 is not too bad...?

- Measurement technique

- Assumptions in empirical formulation 


\section{Simulation Validation- Stress states}

\section{XRD Scan NTO2}

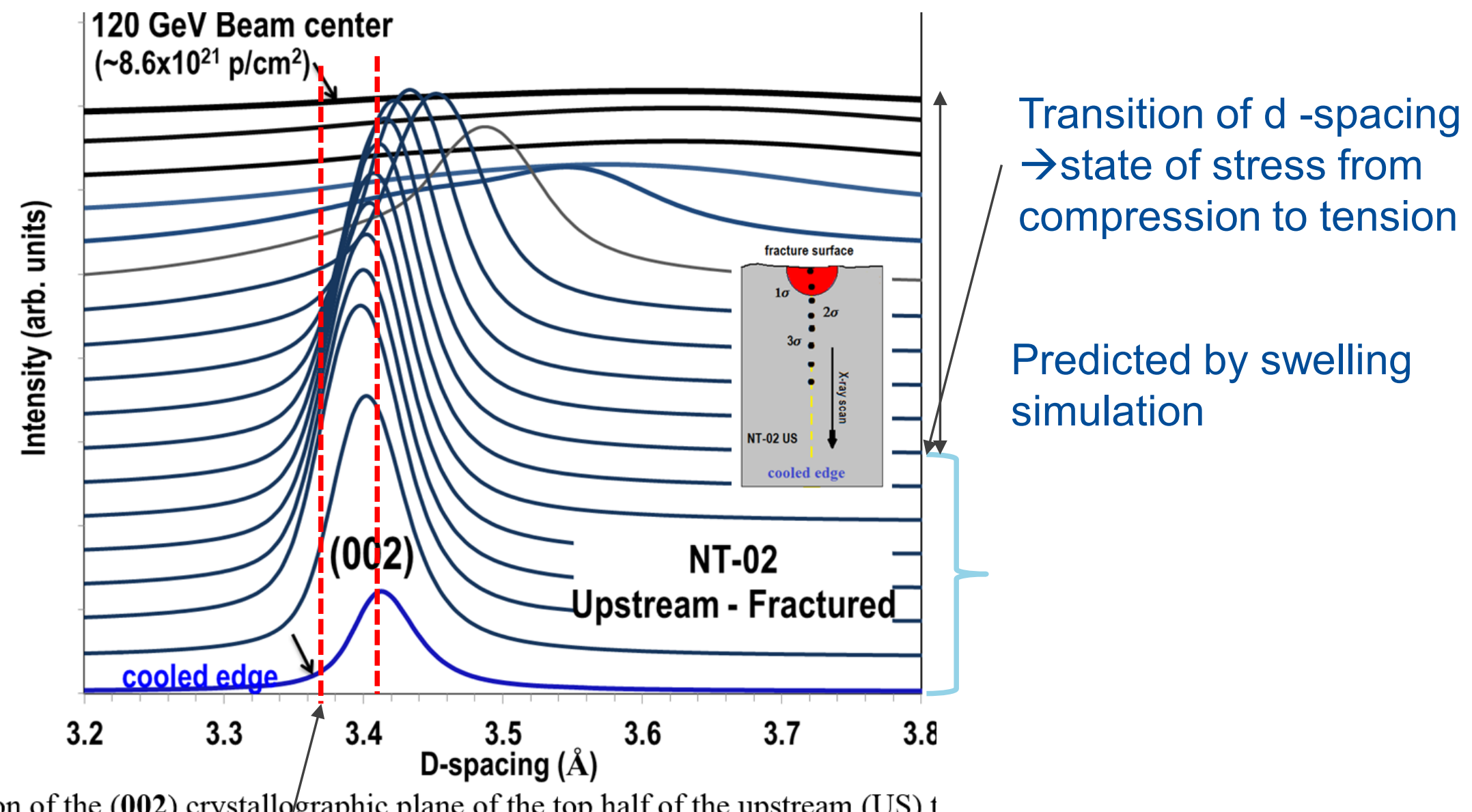

In of the (002) crystallographic plane of the top half of the upstream (US) 1

Undeformed crystallite d-spacing 3.37 


\section{Simulation Validation- Mohr-Coulumb's factor of safety}

FS distribution

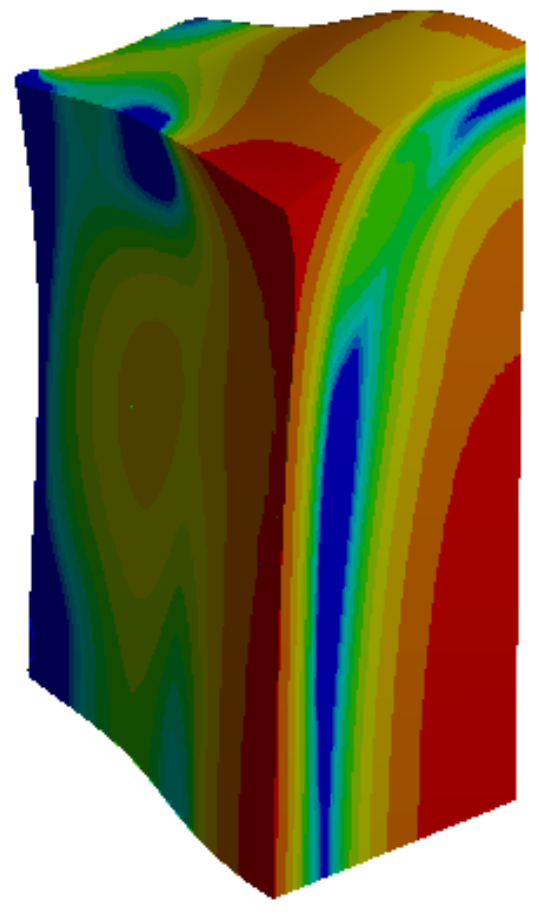

FS $<1$

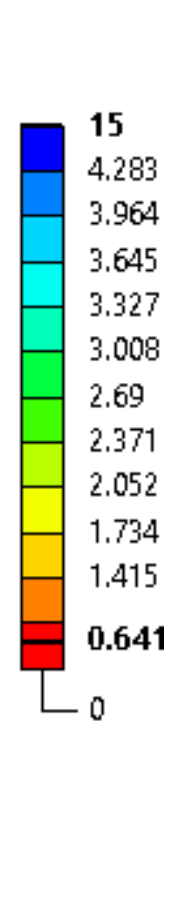

$$
F_{\text {safety }}=\left(\frac{\sigma_{1}}{\sigma_{u t}}+\frac{\sigma_{3}}{\sigma_{u c}}\right)^{-1}
$$

Probable crack initiation sites

Fractography suggest crack starting from inside and propagating outward 


\section{Dynamic stresses- $\sigma_{\mathrm{yy}}$}

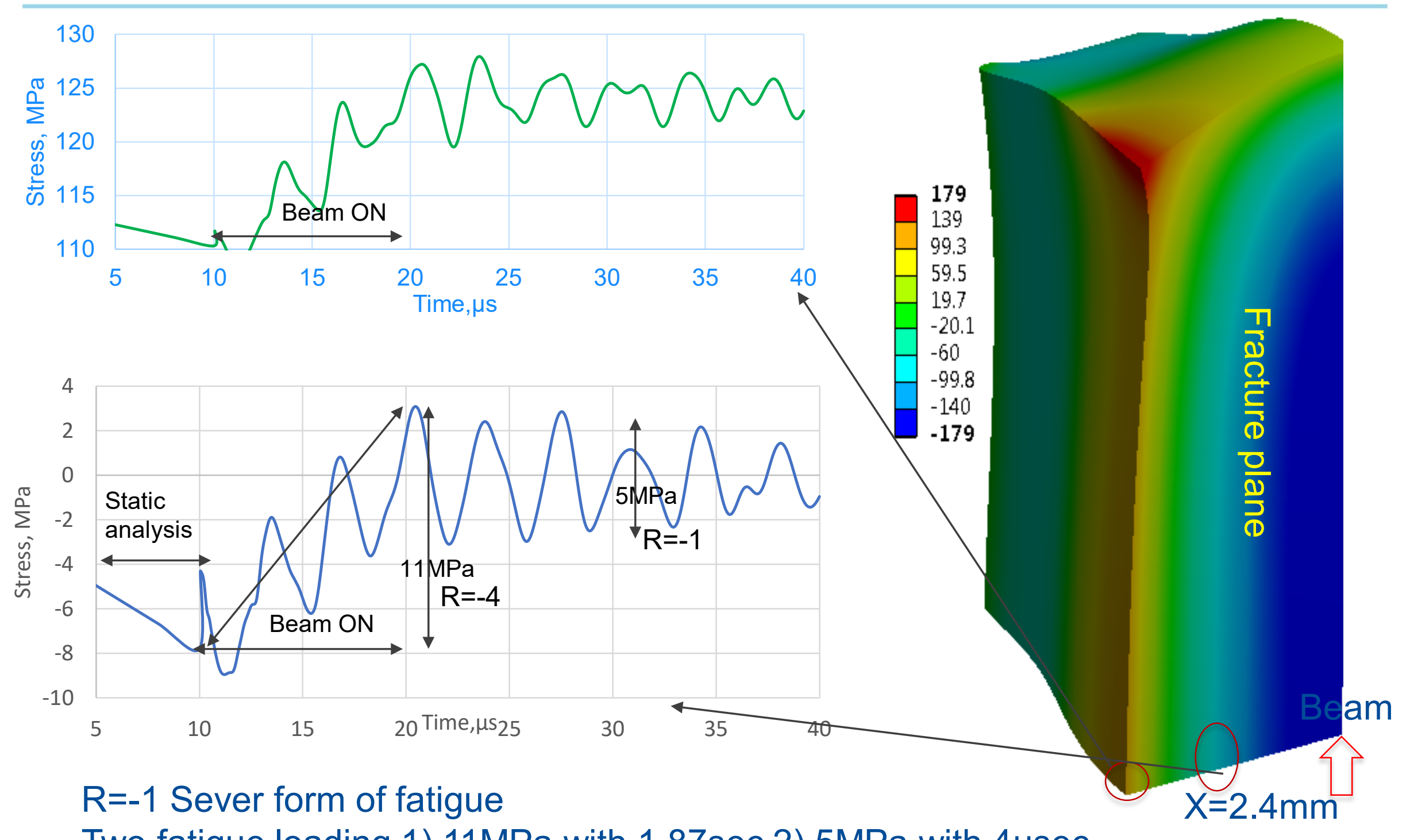

Two fatigue loading 1) $11 \mathrm{MPa}$ with $1.87 \mathrm{sec} 2$ ) $5 \mathrm{MPa}$ with $4 \mu \mathrm{sec}$ 


\section{Fatigue Crack growth stages}

Crack initiation $\rightarrow$ significant portion of fatigue life

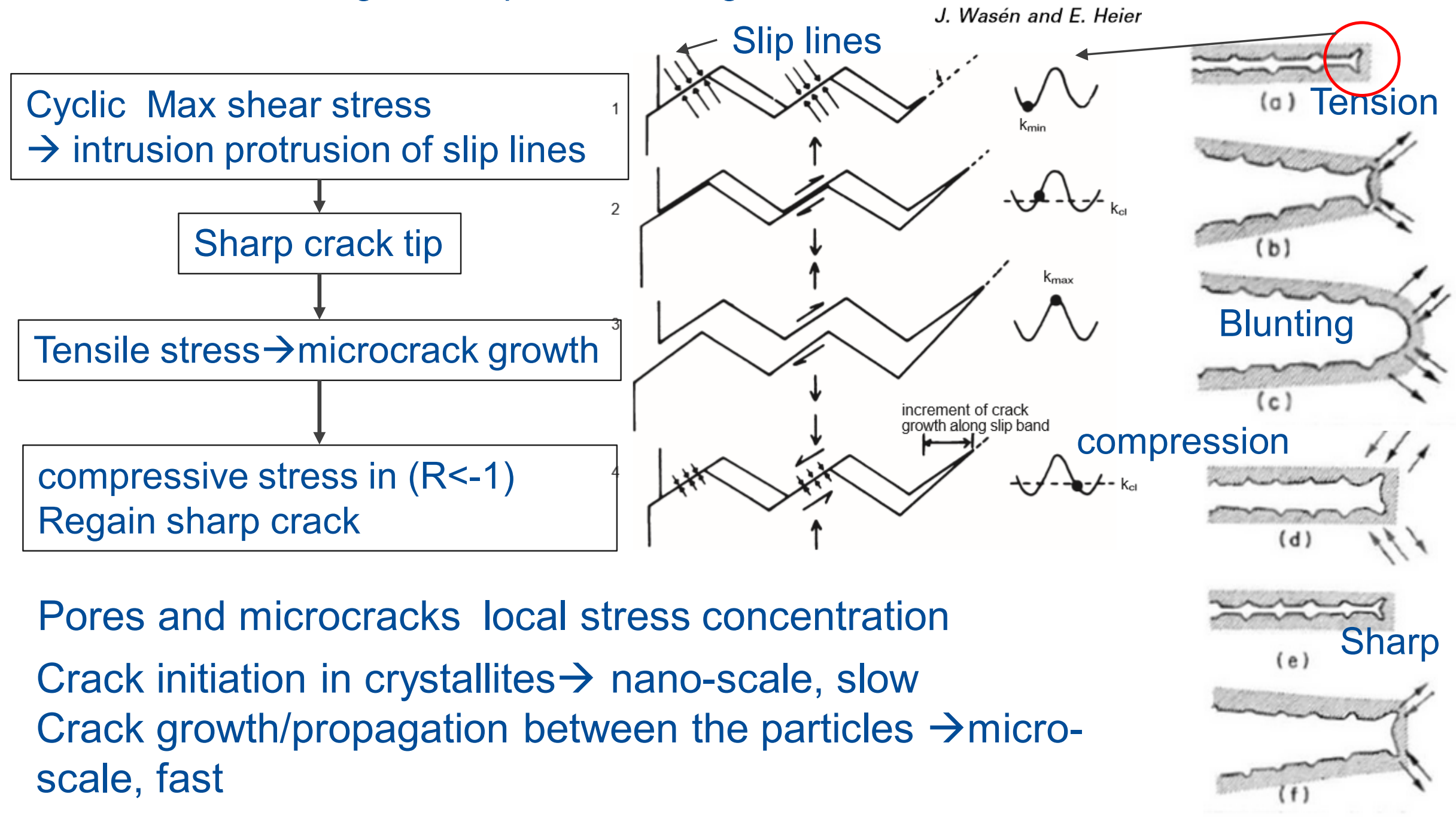




\section{Conclusions}

- Thermal stresses alone could not have caused crack

- Implementing swelling strain effectively raised the stress state close to failure strength.

- Simulation could explain probable crack initiation region

- Regions with Factor of safety $<1$

- Dynamic stresses due to beam heating $\rightarrow$ potential fatigue crack

- High amplitude low frequency $\rightarrow 11 \mathrm{Mpa} 15$ million cycles per year

- Low amplitude high frequency $\rightarrow 5 \mathrm{Mpa}$ Gigacycles per year

- Regions of complete load reversal at about $2.45 \mathrm{~mm}$ from beam center

- Graphite has low endurance limit 14 Mpa (@1million cycle

- Low amplitude gigacycle load can cause fatigue failure 


\section{Summary}

- Developed an empirical formula for local swelling (Temp, fluence)

- Simulation results bulk swelling reasonable agreement with measurement

- Combined swelling stress and dynamic stress due to beam loading caused failure

Did not take into account

- Stress, creep effect on swelling.

- Dislocation distribution and type, grain boundary segregation etc.

In Future $\rightarrow$ Make the model predictive

- Obtain parameters from mechanism of swelling rather than XRD 


\section{Thank You!}

